\title{
CONSTRUCCIÓN CONCEPTUAL DE LA COMPETENCIA GLOBAL EN EDUCACIÓN
}

\author{
Conceptual Construction of Global Competence \\ in Education
}

\author{
María SANZ LEAL, Martha Lucía OROZCO GÓMEZ y Radu Bogdan TOMA \\ Universidad de Burgos. España. \\ msleal@ubu.es;mlorozco@ubu.es; rbtoma@ubu.es \\ https://orcid.org/0000-0002-9845-0688; https://orcid.org/0000-0001-5547-8712; \\ bttps://orcid.org/0000-0003-4846-7323
}

Fecha de recepción: 04/01/2021

Fecha de aceptación: 03/04/2021

Fecha de publicación en línea: 01/01/2022

Cómo citar este artículo: Sanz Leal, M. a, Orozco Gómez, M. L., y Toma, R. B. (2022). Construcción conceptual de la competencia global en educación. Teoría de la Educación. Revista Interuniversitaria, 34(1), 83-103. https://doi.org/10.14201/teri.25394

\section{RESUMEN}

La competencia global como objetivo de aprendizaje ha tomado relevancia desde su inclusión en el Programa para la Evaluación Internacional de los Estudiantes (PISA). Pese al creciente interés por esta competencia, son numerosas las cuestiones que requieren de una reflexión profunda, tales como ¿Qué es la competencia global? ¿Cómo se ha construido? ¿Desde qué enfoques se parte en los procesos discursivos para su construcción? y ¿Qué aporta la educación intercultural a este constructo? Para dar respuesta a estas preguntas, este artículo analiza los antecedentes, las conceptualizaciones, enfoques y teorías en base a las cuales se ha construido este concepto, haciendo especial énfasis en la interculturalidad como enfoque y modelo educativo que favorece la inclusión. El método utilizado es una revisión crítica y un análisis discursivo. Se ha encontrado que en la conceptualización ha predominado el enfoque 
instrumentalista de eficiencia social, aunque sea de manera subyacente, frente a un enfoque de re-construccionismo social que suele aparecer en primer plano. En muchas ocasiones los constructos competencia global y competencia intercultural se utilizan indistintamente, si bien la primera resulta más integral para afrontar los desafíos de la globalización. Por último, se señalan desafíos y limitaciones conceptuales y de medición que dificultan la generalización y comparación entre estudios a nivel internacional de la adquisición de esta competencia y que requieren por tanto de más estudios que investiguen sistemáticamente la construcción del concepto, su desarrollo y medición.

Palabras clave: teoría de la educación; competencia global; educación intercultural; educación para la ciudadanía global; educación basada en competencias; educación transformadora.

\section{ABSTRACT}

Global competence as a learning objective has become relevant since its inclusion in the Programme for International Student Assessment (PISA). Despite the growing interest in this competence, there are several issues requiring deep reflection, such as: What is global competence? How has it been constructed? From which approaches are the discursive processes for its construction based? and What does intercultural education contribute to this construct? To answer these questions, this paper analyses the background, conceptualisations, approaches and theories on which the concept has been built, with special emphasis on interculturality as an educational approach and model that favours inclusion. The method used is a critical review and discursive analysis. It has been found that the instrumentalist approach to social efficiency has predominated in the conceptualization, (at least as the underlying approach), as opposed to an approach of social re-constructionism that usually appears in the foreground. On many occasions the constructs global competence and intercultural competence are used interchangeably, although the former is more comprehensive in addressing the challenges of globalisation. Finally, it is highlighted that there are conceptual and measurement challenges and limitations that make difficult comparing the acquisition of this competence at an international level and that therefore require more studies that systematically investigate the construction of the concept, its development and measurement.

Key words: educational theories; global competence; intercultural education; global citizenship education; competence-based education; transformative education.

\section{INTRODUCCIÓN}

El actual contexto mundial evidencia el grado de interconexión e interdependencia entre países, culturas y sociedades del mundo. Quizá, la pandemia por la enfermedad del coronavirus permita evidenciar estas interdependencias globales y la vulnerabilidad de los estados. Asimismo, las crisis migratorias y de refugiados, el 
calentamiento global y su consecuente cambio climático, las crisis sanitarias o las crisis financieras reflejan los fenómenos relacionados con los efectos de la globalización que enfrentamos en el siglo XXI. La sostenibilidad medioambiental, la diversidad cultural, reducir la desigualdad y la pobreza son los actuales desafíos que marcan la agenda del presente y a los que la educación debe dar respuesta. La Agenda 2030 para el Desarrollo Sostenible indica que todas las acciones educativas deberían estar en conexión con la Meta 4.7, de los Objetivos de Desarrollo Sostenible:

Asegurar que todos los alumnos adquieran los conocimientos teóricos y prácticos necesarios para promover el desarrollo sostenible, entre otras cosas mediante la educación para el desarrollo sostenible y los estilos de vida sostenibles, los derechos humanos, la igualdad de género, la promoción de una cultura de paz y no violencia, la ciudadanía mundial y la valoración de la diversidad cultural y la contribución de la cultura al desarrollo sostenible (UNESCO, 2015, p. 22)

Para responder a estos desafíos provocados por la globalización, han ido apareciendo diferentes modelos de educación global, ${ }^{1}$ como la educación para la ciudadanía global (ECG), la educación democrática, la educación para el desarrollo sostenible (EDS) o la educación intercultural. Aunque estos modelos tengan diferencias en su enfoque y alcance (cosmopolitismo, promoción de los derechos humanos y participación activa, sostenibilidad ambiental o diversidad cultural), comparten un objetivo común para fomentar la comprensión del mundo, las relaciones positivas y una participación activa y transformadora en y para la sociedad.

La EDS y ECG, facilitan la comprensión de las interrelaciones económicas, sociales, culturales y políticas entre el Norte y el Sur global, promueven valores y actitudes relacionados con la solidaridad y la justicia social. La primera se centra más en alcanzar un desarrollo humano y sostenible y la segunda en promover personas responsables y activas. La educación intercultural por su parte se centra en favorecer interacciones positivas y enriquecedoras entre individuos de culturas diversas y la educación democrática toma de referencia la Declaración de los Derechos Humanos y la participación activa y en igualdad de los individuos en la toma de decisiones. En este artículo nos centramos en los aportes de la educación intercultural porque creemos que en las escuelas donde más se materializa y se puede ver de cerca los efectos de la globalización es en la diversidad cultural cada vez mayor en las aulas, siendo una oportunidad para establecer relaciones con todas las dimensiones de la competencia global que se verán más adelante.

A su vez, el surgimiento del enfoque basado en competencias (EBC) motivado entre otras cosas, por la necesidad de desarrollar capacidades amplias en las personas que les permitan adecuarse a situaciones cambiantes ha generado la aparición de

1. Global no es sinónimo de general sino de fenómeno contemporáneo relacionado con la globalización. 
conceptualizaciones como la competencia intercultural, la competencia democrática o la competencia global, ligadas a esos modelos de educación global.

La Organización para la Cooperación y el Desarrollo Económicos (OCDE) a través de PISA evalúa el nivel del alumnado cuando llegan al final de la etapa de enseñanza obligatoria y cada año incluye un área innovadora, en 2018 fue la competencia global. Esta organización define la competencia global como: «la capacidad de analizar asuntos globales e interculturales, valorar distintas perspectivas desde el respeto por los derechos humanos, para interrelacionarse con personas de diferentes culturas emprender acciones por el bien común y por el desarrollo sostenible» (OCDE, 2018, p. 5).

Las teorías recientes del «nuevo institucionalismo sociológico» señalan que existen mecanismos globales de influencia en las políticas educativas locales (imposición, armonización, difusión, estandarización e instalación de interdependencia) en los que las organizaciones internacionales son transmisoras de ideas de educación global, que suelen tener un carácter instrumental y orientado al mercado (Hajisoteriou \& Angelides, 2016, citado en Hajisoteriou \& Angelides 2020, p. 150). La estandarización se lleva a cabo a través de procedimientos de evaluación globalizados, tales como pruebas de desempeño internacionales como PISA.

En el contexto español en el que se plantea el estudio, apenas se ha teorizado sobre el concepto de competencia global, siendo la educación intercultural y la educación para el desarrollo y la ciudadanía global² los más desarrollados, al igual que ocurre por ejemplo en Alemania donde la competencia global es discutida especialmente respecto a la educación para el desarrollo global y sostenible (Sälzer \& Roczen, 2018). El informe del Ministerio de Educación y Formación Profesional (MEFP, 2020) expone los resultados de la evaluación en competencia global de 2018, para el que, en términos generales en España, el 68\% del alumnado alcanza o supera el nivel básico de rendimiento en competencial global.

En este contexto surgen varias preguntas de investigación tales como, ¿qué es la competencia global? ¿cómo se ha construido? ¿desde qué enfoques se parte en los procesos discursivos para su construcción? y ¿qué aporta la educación intercultural a este constructo?

A través de este trabajo, se pretende dar respuesta a estas preguntas, proponiendo un marco conceptual que permita seguir investigando sobre la competencia global, su desarrollo y evaluación. Para ello se han tenido en cuenta los antecedentes, las conceptualizaciones y las teorías que se consideran válidas para el correcto encuadre del estudio como recomiendan Hernández et al. (2010), de manera que

2. La educación para el desarrollo sostenible y la ciudadanía global son promovidas sobre todo en el ámbito de la cooperación internacional para el desarrollo por ONGD y centros de cooperación. La educación intercultural más desarrollada en el ámbito de la educación formal se centra en la inclusión educativa en entornos de diversidad. 
ayuden a conceptualizar qué se entiende por competencia global y qué aspectos se relacionan con una educación inclusiva desde un enfoque de interculturalidad.

En definitiva, se explica cómo se ha construido la competencia global, desde qué enfoques y para qué fines, qué desafíos y limitaciones conlleva y qué futuras líneas de investigación se consideran necesarias.

\section{ANTECEDENTES TEÓRICOS}

La educación basada en competencias ha implicado un cambio profundo en la forma de entender la educación. En recientes revisiones se sitúa su aparición entre finales de los años 80 y mediados de los años 90 donde tiene su verdadero impulso con el informe que lideró Delors et al. (1996) para la UNESCO, después el proyecto Definición y Selección de Competencias (DeSeCo) de la OCDE (2005) identifica y define las competencias clave necesarias para las generaciones futuras y su capacidad para manejar un mundo que cambia rápidamente.

Pocos estudios presentan definiciones propias del término competencia y la mayoría adoptan marcos teóricos ya consolidados, gran parte se adhieren al marco teórico propuesto por la OCDE que se utiliza como una fuente autorizada que no se cuestiona, analiza o amplía (García, 2008; Ortiz-Revilla et al., 2020; Tahirsylaj \& Sundberg, 2020).

El marco DeSeCo plantea tres amplias categorías de competencias clave: actuar de forma autónoma, utilizar herramientas de forma interactiva y funcionar en grupos socialmente heterogéneos; esta última categoría tiene mucha influencia en la competencia global como se verá más adelante. La Comisión Europea se basó en el proyecto DeSeCo y el propuesto por la UNESCO en su recomendación conjunta del Parlamento Europeo y del Consejo (2006) sobre las competencias clave para el aprendizaje permanente (2006/962/CE), contribuyendo a su vez con la definición de ocho competencias clave recomendadas para su adopción dentro de la Unión Europea, algunas de ellas como la comunicación en lenguas extranjeras, competencias sociales y cívicas o conciencia y expresión cultural muy relacionadas con la competencia global.

En la investigación educativa la definición genérica de competencia integra conocimientos, habilidades y actitudes (y a veces valores) orientadas a la acción, siendo así como se define la competencia global. También indica que la investigación sobre las competencias en educación se dirige en su mayoría a la generalidad competencial y cuando se especifica sobre una materia, se muestra que las ciencias, las matemáticas y las tecnologías están muy sobrerrepresentadas (García, 2008; Ortiz-Revilla et al. 2020; Tahirsylaj \& Sundberg, 2020). 


\section{Metodología}

Atendiendo a la clasificación de los tipos de revisiones de la literatura propuesta por Grant y Booth (2009), el presente estudio se enmarca en las revisiones críticas en tanto: (i) la búsqueda no es exhaustiva, (ii) presenta los resultados de manera narrativa y cronológica, y (iii) del análisis de los estudios se realiza una contribución conceptual a la teoría existente. En este tipo de revisión se busca identificar lo que se ha logrado anteriormente y se suele aplicar para debates generales y con el fin de suplir una falta de conocimiento sobre un tema. En este caso, la ausencia de reflexión y debate sobre la competencia global en el contexto español, permitiendo la consolidación y facilitando un trabajo previo a futuros investigadores.

Para evitar la posibilidad de sesgo con la que cuenta este tipo de revisión algunos autores plantean la necesidad de exponer el proceso de búsqueda (Guirao Goris, 2015). En este sentido, esta revisión crítica de la literatura recoge el origen del concepto y los marcos más recientes que, en algunos casos, se encuentran en documentos institucionales que no aparecen en bases de datos lo que motiva la elección de este método. La búsqueda se realizó durante los meses de mayo y junio de 2020 y se consultaron las bases de datos SCOPUS, Web of Science y Dialnet empleando el descriptor "global* competen*" y su correspondiente denominación en castellano. Se empleó el símbolo * (asterisco) como operador de truncamiento para buscar diferentes variantes del término globally/global, competencias/competence. Se seleccionaron documentos publicados en inglés y español desde 2001 al 2020. Se completó la búsqueda con la lectura y rastreo de bibliografía referenciada en los documentos seleccionados que permitiesen un análisis cronológico característico de este tipo de revisión (Grant \& Booth, 2009, p. 94).

\subsection{Análisis del discurso}

Explorar y mapear la forma en la que ha sido enmarcada y definida la competencia global requiere de un marco teórico que permita diferenciar los distintos enfoques en los procesos discursivos para su construcción. Tahirsylaj \& Sundberg (2020) plantean que, para diferenciar los distintos aspectos para la conceptualización de las competencias, es necesario examinar, además de las definiciones explícitas de competencias, los supuestos implícitos que se dan por sentado. Estos autores llaman a esos supuestos, "las ideas de fondo", y a las definiciones explícitas las denominan «ideas de primer plano».

Las ideas de fondo son principios básicos que se dan por sentado e institucionalizados, y rara vez se cuestionan, excepto en tiempos de crisis. Las 'ideas de primer plano', por otro lado, son las ideas y conceptos que estructuran los programas educativos y las reformas en la formulación de políticas. Estas ideas y definiciones se utilizan en la interacción discursiva para mantener y / o alterar las instituciones y sus patrones de acción. (Schmidt, 2015, citado en Tahirsylaj \& Sundberg, 2020, p. 133) 
Tahirsylaj \& Sundberg (2020) plantean que las ideas de fondo que enmarcan las definiciones más comúnmente utilizadas de EBC tienen un patrón claro donde los paradigmas dominantes que influyen en las definiciones se relacionan principalmente con la eficiencia social y la teoría del capital humano (Deng \& Luke, 2008). Las competencias se enmarcan como los recursos más importantes y fundamentales para que las personas se adapten a la economía del conocimiento. Sin embargo, las ideas dominantes en primer plano, es decir, las que se explicitan para definir el EBC, no provienen de la teoría del capital humano, sino de la ideología del currículo centrado en el alumno. Dicho de otro modo, existe una tendencia a asumir las competencias desde una mirada técnica o profesionalizante, aunque se presenten desde referencias y argumentos teóricos de visión humanista y formadora (OrtizRevilla et al. 2020; Tahirsylaj \& Sundberg, 2020).

Esas conclusiones resultan de contrastar los estudios con los cuatro paradigmas curriculares principales con influencia histórica y actual en los propósitos de la escolarización que plantean Deng \& Luke (2008) y Schiro (2013): el racionalismo académico, que da importancia a la transmisión de conocimientos disciplinarios; el re-construccionismo social, donde el propósito de la educación es facilitar la justicia social para permitir acabar con las desigualdades; la eficiencia social, para la productividad económica y social que permita la perpetuación de la sociedad; y finalmente, la ideología curricular centrada en el alumno, cuyo foco no son las disciplinas académicas o las necesidades de la sociedad, sino las necesidades e intereses de los individuos para promover su desarrollo personal y auto-realización (humanismo).

En los siguientes apartados se exploran las 'ideas de fondo' y las 'ideas de primer plano' de documentos que han aportado definiciones consolidadas para la competencia global a la luz de esos cuatro paradigmas, tal como indica la lógica de investigación y categorías previas en el análisis del discurso establecidas por Santander (2011, p. 214).

\section{CONSTRUYENDO LA COMPETENCIA GLOBAL}

La conceptualización de la competencia global se ha desarrollado mayoritariamente en EE. UU. y por instituciones supranacionales como la OCDE o la UNESCO. En el caso de España las referencias encontradas a este concepto relacionadas con la educación en general son muy recientes y utilizan las definiciones propuestas por el marco de PISA 2018, como Cornejo y Gómez-Jarabo (2018) y Heras et al. (2019) o la propuesta por Boix-Mansilla \& Jackson (2013) en el trabajo de GarcíaBeltrán et al. (2019).

El reto de definir la competencia global ha ido de la mano de la rápida, creciente y crítica necesidad de los colegios y universidades de internacionalizarse (Auld \& Morris, 2019; Hunter et al., 2006; Kim, 2019). La educación internacional ${ }^{3}$ es vista

3. En EE. UU. ya en la década de 1960 las universidades contaban con estudiantes universitarios extranjeros, en Europa el auge comenzaría a finales de los años 80 con el Programa Erasmus. 
como precursora de la creación y fomento de la competencia global a través de la movilidad internacional, los programas de intercambio, el aprendizaje de idiomas o el fomento del bilingüismo (Lambert, 1993).

Reimers (2010) considera que la competencia global ha sido desarrollada hasta el momento en familias, escuelas y universidades de las élites a través del dominio de idiomas extranjeros y temas globales para especialistas en estudios de área y que esas competencias son necesarias para la mayoría de la población mundial, no solo para unos pocos, «la competencia global debería ser ahora un propósito de la educación de masas, no sólo de la educación de élite» (Reimers, 2010, p. 186).

\subsection{Origenes del concepto}

Según Hunter (2004) la competencia global fue mencionada por primera vez en 1988 en una iniciativa de educación internacional publicada en un informe del Council on International Education Exchange de EE. UU. de América. En los años 90 se promueven varias conferencias internacionales dedicadas a definir la competencia global desde el ámbito de las políticas educativas, a través de sus informes podemos extraer como se fue construyendo este concepto. En el informe de 1993 se comenzaban a vislumbrar algunos de los enfoques de la competencia global, sus dimensiones y algunas propuestas para su definición. Dicho informe expone que abordar la competencia global puede hacerse desde el punto de vista de los intercambios internacionales, la educación para la ciudadanía, la diversidad y el multiculturalismo o la formación de especialistas y profesorado de estudios de idiomas (Lambert, 1993).

Richard D. Lambert, considerado como el padre de la competencia global, habla de una cualidad personal especial que también se ha llamado conciencia o perspectiva global o cosmopolita y establece cinco componentes: conocimiento (de eventos actuales), empatía, aprobación (actitud positiva), competencia en lenguas extranjeras y desempeño de tareas (capacidad para comprender el valor en algo ajeno).

La relación entre la competencia global y la vida empresarial y laboral es una constante, el informe "Educar para la competencia global. Pasaporte de Estados Unidos para el futurom de 1998 es más explícito, expone la competencia global como educación superior para el desarrollo de recursos humanos para una economía mundial y el liderazgo de los EE. UU. Su objetivo es demostrar la importancia de la cooperación internacional en materia de educación y desarrollo para el futuro económico y político de la nación e incentivar la asociación entre gobiernos, empresas y universidades para incluir en los planes de estudios una dimensión internacional que prepare al profesorado y a una fuerza de trabajo capaz de liderar y competir en los mercados mundiales (American Council on Education, 1998). Aunque también se menciona la sostenibilidad medioambiental, la preocupación sobre los refugiados o la defensa de los derechos humanos, se hace desde la visión de amenaza a la prosperidad y seguridad de los Estados Unidos. 


\subsection{Definiciones, marcos conceptuales y propuestas de evaluación}

En la Tabla 1. se recogen las definiciones más utilizadas junto a sus autores y los enfoques que se consideran más significativos para cada una de las fuentes, en base a los cuatro paradigmas con influencia histórica y actual en los propósitos de la escolarización explicados ya en el apartado de metodología. La categorización de dichos enfoques y de lo que se ha considerado 'ideas de primer plano' o 'ideas de fondo' se ha realizado a través del análisis crítico del discurso de los documentos referenciados, siguiendo las indicaciones de Santander (2011, p. 216) en las que la unidad de análisis es la oración. Es por ello que en el análisis posterior en algunos casos se utilizan expresiones literales de los documentos para ejemplificar su enfoque.

TABLA 1

IDEAS DE PRIMER PLANO VS IDEAS DE FONDO EN LA COMPETENCIA GLOBAL

\begin{tabular}{|c|c|c|c|}
\hline FUENTE & DEFINICIÓN & $\begin{array}{l}\text { Ideas de } \\
\text { Fondo }\end{array}$ & $\begin{array}{c}\text { Ideas de } \\
\text { primer plano }\end{array}$ \\
\hline $\begin{array}{l}\text { (American } \\
\text { Council on } \\
\text { International } \\
\text { Intercultural } \\
\text { Education, } \\
\text { 1996) }\end{array}$ & $\begin{array}{l}\text { Capacidad de comprender la interco- } \\
\text { nexión de pueblos y sistemas, tener un } \\
\text { conocimiento general de acontecimien- } \\
\text { tos mundiales, aceptar y hacer frente a la } \\
\text { existencia de diferentes valores y actitu- } \\
\text { des culturales y celebrar la riqueza y los } \\
\text { beneficios de esta diversidad. (p. 2). }\end{array}$ & $\begin{array}{c}\text { Eficiencia } \\
\text { social }\end{array}$ & $\begin{array}{l}\text { Re-construcción } \\
\text { social }\end{array}$ \\
\hline $\begin{array}{l}\text { (Olson \& } \\
\text { Kroeger, } \\
\text { 2001) }\end{array}$ & $\begin{array}{l}\text { "Tener suficiente conocimiento sustanti- } \\
\text { vo, comprensión perceptiva y habilidades } \\
\text { de comunicación intercultural para inte- } \\
\text { ractuar eficazmente en nuestro mundo } \\
\text { globalmente interdependiente» (p. 117). }\end{array}$ & $\begin{array}{c}\text { Re- } \\
\text { construcción } \\
\text { social }\end{array}$ & $\begin{array}{l}\text { Re-construcción } \\
\text { social }\end{array}$ \\
\hline $\begin{array}{l}\text { (Hunter, } \\
\text { 2004) }\end{array}$ & $\begin{array}{l}\text { «Tener una mente abierta mientras se } \\
\text { busca activamente entender las normas } \\
\text { culturales y las expectativas de los demás, } \\
\text { aprovechando este conocimiento adquiri- } \\
\text { do para interactuar, comunicar y trabajar } \\
\text { eficazmente fuera de su entorno" (p. 101). }\end{array}$ & $\begin{array}{l}\text { Eficiencia } \\
\text { social }\end{array}$ & $\begin{array}{l}\text { Re-construcción } \\
\text { social }\end{array}$ \\
\hline $\begin{array}{l}\text { (Reimers, } \\
\text { 2010) }\end{array}$ & $\begin{array}{l}\text { "El conocimiento y las habilidades para ayu- } \\
\text { dar a las personas a comprender el mundo } \\
\text { plano en el que viven, integrarse a través } \\
\text { de los dominios disciplinarios para com- } \\
\text { prender los asuntos y eventos globales, y } \\
\text { crear posibilidades para abordarlos. Son } \\
\text { también las disposiciones actitudinales y éti- } \\
\text { cas que hacen posible interactuar pacífica, } \\
\text { respetuosa y productivamente con los seres } \\
\text { humanos de diversas geografías" (p. 184). }\end{array}$ & - & $\begin{array}{l}\text { Re-construcción } \\
\text { social }\end{array}$ \\
\hline
\end{tabular}




\begin{tabular}{|c|c|c|c|}
\hline FUENTE & DEFINICIÓN & $\begin{array}{l}\text { Ideas de } \\
\text { Fondo }\end{array}$ & $\begin{array}{c}\text { Ideas de } \\
\text { primer plano }\end{array}$ \\
\hline $\begin{array}{l}\text { (Boix- } \\
\text { Mansilla } \\
\text { \& Jackson, } \\
\text { 2011) }\end{array}$ & $\begin{array}{l}\text { "Es la capacidad y la disposición para } \\
\text { comprender y actuar en cuestiones de } \\
\text { importancia mundial» (p. 13). }\end{array}$ & $\begin{array}{l}\text { Eficiencia } \\
\text { social }\end{array}$ & $\begin{array}{c}\text { Re-construcción } \\
\text { social }\end{array}$ \\
\hline $\begin{array}{l}\text { (OCDE, } \\
\text { 2014) }\end{array}$ & $\begin{array}{l}\text { "La capacidad de un individuo para com- } \\
\text { prender que aprendemos, trabajamos y } \\
\text { vivimos en un ambiente internacional, so- } \\
\text { ciedad interconectada e interdependiente } \\
\text { y la capacidad de utilizar ese conoci- } \\
\text { miento para informar las disposiciones, } \\
\text { comportamientos y acciones de cada uno } \\
\text { al interactuar, comunicarse participan- } \\
\text { do en una variedad de roles y contextos } \\
\text { internacionales como un individuo re- } \\
\text { flexivo" (p. 9). }\end{array}$ & $\begin{array}{l}\text { Eficiencia } \\
\text { social }\end{array}$ & $\begin{array}{c}\text { Re-construcción } \\
\text { social }\end{array}$ \\
\hline $\begin{array}{l}\text { (OCDE, } \\
\text { 2018) }\end{array}$ & $\begin{array}{l}\text { "Es un objetivo de aprendizaje multidi- } \\
\text { mensional y permanente. Los individuos } \\
\text { competentes a escala mundial pueden } \\
\text { examinar cuestiones locales, globales e } \\
\text { interculturales, comprender y apreciar } \\
\text { diferentes perspectivas y visiones del } \\
\text { mundo, interactuar con éxito y de mane- } \\
\text { ra respetuosa con los demás y actuar de } \\
\text { modo responsable hacia la sostenibilidad } \\
\text { y el bienestar colectivo» (p. } 5 \text { ). }\end{array}$ & $\begin{array}{l}\text { Eficiencia } \\
\text { social }\end{array}$ & $\begin{array}{c}\text { Re-construcción } \\
\text { social }\end{array}$ \\
\hline $\begin{array}{l}\text { (Boix- } \\
\text { Mansilla } \\
\text { \& Wilson, } \\
\text { 2020) }\end{array}$ & $\begin{array}{l}\text { «El proceso de por vida de cultivarse a } \\
\text { uno mismo, la capacidad humana y la dis- } \\
\text { posición para comprender los problemas } \\
\text { de importancia global y cultural y actuar } \\
\text { para el bienestar colectivo y el desarrollo } \\
\text { sostenible» (p. 11). }\end{array}$ & - & $\begin{array}{l}\text { Enfoque } \\
\text { centrado en el } \\
\text { alumno }\end{array}$ \\
\hline
\end{tabular}

Fuente: Elaboración propia

Olson y Kroeger (2001) relacionan la adquisición de una segunda lengua, la experiencia en el extranjero y la sensibilidad intercultural para evaluar la competencia global. Entienden que nuestras decisiones laborales y de vida son de naturaleza global y prácticamente toda interacción humana es un encuentro intercultural. Aportan un enfoque más centrado en aspectos de la competencia intercultural guiados por el modelo de sensibilidad intercultural de Bennett ofreciendo una propuesta de definición de la competencia global que empieza a dejar entrever como la ésta se construye por varias dimensiones, en las cuales las relaciones interculturales tienen 
un importante peso «... conocimiento sustantivo, comprensión perceptiva, y habilidades de comunicación intercultural...»(Olson \& Kroeger, 2001, p. 117).

Hunter (2004) a través de un panel Delphi internacional ofrece una definición que también incluye aspectos relacionados con la competencia intercultural. Cita a autores como Wilson y Dalton (1997) y Curran (s. f.), que dotan la competencia global de la comprensión perceptiva, que se refiere a aspectos relacionados con una mentalidad abierta que busca activamente comprender las normas culturales y las expectativas de los demás resistiendo a los estereotipos.

Este autor además de ofrecer una conceptualización también busca comprobar si la perspectiva sobre competencia global de los reclutadores de empresas multinacionales y los educadores universitarios coinciden, mostrando un claro enfoque de eficiencia social; "...lo que me llevó a buscar unir a los educadores y aquellos que necesitan su producto (empresarios)» (Hunter, 2004, p. 104).

Fernando Reimers, hace la primera propuesta de que la OCDE debería evaluar las competencias globales (Auld \& Morris, 2019). Este autor considera que las ventajas económicas que se derivan de la competencia mundial han recibido más atención que las ventajas cívicas, cuando la competencia global es útil no solo desde el punto de vista económico sino como piedra angular del liderazgo democrático y la ciudadanía. Concibe la competencia global como un conjunto de conocimientos y habilidades que permiten a las personas comprender el mundo y sus desafíos de manera que puedan crear posibilidades para abordarlos, además de una disposición actitudinal y ética que permiten interacciones pacíficas, respetuosas y productivas entre personas de diversas geografías. Conjuga tres dimensiones: afectiva, de acción y académica, especificando en distintos aspectos como: desarrollo del carácter, el afecto y los valores; las aptitudes y el desarrollo de la motivación para actuar; y un aspecto basado en el desarrollo de la cognición, conocimiento y capacidad para comprender los problemas mundiales (Reimers, 2010, p. 185).

Reimers le da un valor importante al conocimiento y apreciación de los derechos humanos como base común para la primera dimensión de la competencia global, la dimensión ética. Además de insistir en las oportunidades que el alumnado tiene en las propias escuelas para conocer y colaborar con otras personas de diversos orígenes culturales, raciales y socioeconómicos a través de las relaciones entre personal escolar, estudiantes, padres y madres y otros miembros de la comunidad.

Por su parte Boix-Mansilla \& Jackson (2011) ofrecen el marco «Educar para la competencia global: Preparando a nuestra juventud para involucrarse con el mundo" Justifican la necesidad de la competencia global en tres aspectos: La economía global y las demandas cambiantes del trabajo, la migración global con la consecuente diversidad como norma y la inestabilidad climática y gestión ambiental. Afirman que preparar a los jóvenes para el futuro es algo más que para su acceso a la universidad y prepararlos para el trabajo. Entienden que las personas globalmente competentes son conscientes, curiosas e interesadas en aprender sobre el mundo y cómo funciona y establecen cuatro capacidades básicas asociadas con la competencia global: investigar el mundo, reconocer perspectivas, comunicar 
ideas y actuar. Además de una justificación para la educación global y un marco conceptual para la competencia global, desarrollan las cuatro capacidades básicas asociadas y sus relaciones, principios básicos para la enseñanza de la competencia y recomendaciones para que las escuelas e instituciones promuevan una cultura de la competencia global.

Por otro lado, la OCDE en su primer documento clave (OCDE, 2014) relacionado con la competencia global, encarga la definición de un marco para PISA 2018 que cubra las actitudes, comportamientos, conocimientos y habilidades que los estudiantes deberán tener al finalizar la educación para tener éxito en sus futuros estudios y trayectorias laborales. En ese informe se ofrece una definición que irá evolucionando hasta llegar a la definitiva del marco de OCDE (2018). Según Sälzer \& Roczen (2018) la OCDE en 2015 pretendió unir los conceptos de ciudadanía global, competencia intercultural y conocimiento sobre globalización en la idea de competencia global.

Por último, un reciente estudio realizado en escuelas de China aporta una reinterpretación culturalmente informada de la competencia global apoyándose en el modelo de Boix-Mansilla \& Jackson (2011) que parte de un tipo de aprendizaje basado en la capacidad de comprender profundizando en la materia y en la disposición de los estudiantes que tiene que ver con pensamientos y comportamientos globalmente competentes que permitan un aprendizaje duradero (Boix-Mansilla \& Wilson, 2020). La reconceptualización que proponen se basa en la investigación sobre las tradiciones y prácticas chinas en la educación y en las ideas aportadas por profesorado, eso conlleva a incluir el enfoque de oriente que entrelaza la educación con el objetivo de lograr la "autoperfección» dedicada a aprender y cultivar valores, donde el aprendizaje y el conocimiento no están orientados al mundo externo, sino a uno mismo. Consideran la competencia global como un proceso a lo largo de toda la vida en la creación de una persona moral "zuo ren», una brújula moral arraigada en la dignidad humana y la armonía social que informa las acciones individuales y colectivas en el mundo. Por eso incluyen en el marco un elemento natural que simboliza el desarrollo de las capacidades a largo plazo y el cultivo de uno mismo en el centro.

Mayoritariamente las ideas de primer plano encajan en un enfoque de re-construccionismo social y las ideas de fondo se encuadran en un enfoque de eficiencia social, para la productividad económica y social (American Council on Education, 1996, 1998; Boix-Mansilla \& Jackson, 2011; Hunter, 2006; OCDE, 2018). Olson \& Kroeger (2001) por su parte, adoptan un enfoque de fondo de re-construccionismo social centrado en aspectos que influyen en las relaciones entre las personas y BoixMansilla \& Wilson (2020) plantean una aportación que se alinea con una idea en 'primer plano' de la ideología curricular centrada en el alumno, con un enfoque claramente humanista.

Los antecedentes que influyen en la mayor parte de las conceptualizaciones revisadas son la comprensión global evaluada por Barrows et al. (1981), el concepto sobre perspectiva global (conciencia de perspectiva, conciencia sobre el "estado del 
planeta, conciencia transcultural, conocimiento de la dinámica global y conciencia de las elecciones humanas) de Hanvey (1975) y el modelo de sensibilidad intercultural de Bennet (1986).

\section{APORTES DE LA EDUCACión INTERCULTURAL A LA COMPETENCIA GLOBAL}

La educación es intrínsicamente un asunto político, la forma de educar a la ciudadanía se relaciona con el tipo de sociedad que queremos alcanzar. En el ámbito educativo en general la competencia global, en el caso de España, se suele enmarcar como una respuesta a los cambios de la población nacional debido a la inmigración y como resultado de la necesidad de un modelo de ciudadanía más inclusivo (Auld \& Morris, 2019; Cornejo y Gómez-Jarabo, 2018; Goren \& Yemini, 2017).

Como señala Abdallah-Pretceille (2001, p. 57) la heterogeneidad ha sido y es aún considerada un hándicap, fuente de problemas y dificultades en la educación, que justifica medidas compensatorias y segregadoras y las expectativas y creencias del profesorado sobre alumnado de origen inmigrante conllevan en ocasiones a agrupamientos escolares diferenciados (Castejón \& Pàmies, 2018). Existe una fuerte tradición educativa homogeneizadora que ha llevado a concepciones asimilacionistas basadas en ideas etnocéntricas que jerarquizan unas 'culturas' sobre otras y que además lo hacen en base a categorías sociales (nacionalidad, etnia...) que impiden conocer las características individuales y propias de cada persona. Por ello, tanto en Europa como en EE. UU o en Latinoamérica en ocasiones la educación intercultural mal entendida se identifica como el medio para integrar en la cultura de acogida o mayoritaria a los inmigrantes, indígenas u otras etnias. Esa visión ha llevado a prácticas relacionadas con la educación compensatoria, relaciones no igualitarias teñidas de paternalismo o folklorización de las culturas (Aguado, 2003; Deardorff, 2009; Leal, 2016).

Siendo conscientes de esa tradición educativa homogeneizadora que tiene su origen en el modelo educativo taylorista del siglo XIX ligado a producir fuerza de trabajo desde un enfoque de eficiencia social que además predestina al alumnado en base a sus condiciones sociales (origen, clase social, sexo...) se plantea la posibilidad de que la competencia global pueda arrastrar este enfoque. Por ello, se insiste en la necesidad de atender a enfoques en la competencia global que se acerquen más al re-construccionismo social y al humanismo, que permitan fomentar y aumentar las capacidades y potencialidades de cada persona para mejorar su vida siendo agentes activos que transformen la sociedad, contribuyendo con ello a una educación más inclusiva. El enfoque intercultural es propuesto por autores como Aguado y Mata (2017) como mirada que permite comprender la compleja articulación entre diversidad cultural e igualdad en educación. Las diferencias culturales están siempre presentes en todo grupo que se educa, se reconoce la diversidad humana como normalidad, la heterogeneidad como riqueza y la homogeneidad como una forma coercitiva y por tanto contraria a principios democráticos de igualdad. 
El prefijo «inter» de la palabra intercultural es la clave en este paradigma de investigación y acción en educación, donde se establece una relación entre el Yo y el Tu, reconociendo al otro. De acuerdo con Abdallah-Pretceille (2001, p. 39) «el individuo está cada vez menos determinado por la cultura a la que pertenece, no es producto de su cultura, sino, por el contrario, el actor o agente de la misma a través de la interacción». El enfoque intercultural hace hincapié en las relaciones más que en las culturas o los individuos y desde esa perspectiva las diferencias culturales se definen como relaciones dinámicas entre dos entidades que se dan sentido de manera recíproca. Una de las dimensiones de la competencia global se refiere a la comprensión perceptiva, habilidad necesaria en las personas para favorecer esas relaciones de manera positiva.

La comprensión perceptiva no es la única necesaria para que esas relaciones sean lo más igualitarias y enriquecedoras posibles. Aunque haya diferencias en la redacción, existe un consenso sobre las dimensiones reflejadas en la figura 1. que contempla la competencia global, conocimientos, habilidades, actitudes y valores que tienen que ver con el desarrollo cognitivo, socio emocional, actitudinal o conductual y moral-espiritual. Todas las dimensiones están interrelacionadas y tienen una vinculación directa con la educación intercultural. Desde nuestro posicionamiento en enfoques de re-construcionismo social y aprendizaje centrado en el alumno entendemos que favorecen la inclusión educativa.

FIGURA 1

DiMENSIONES DE LA COMPETENCIA GLOBAL

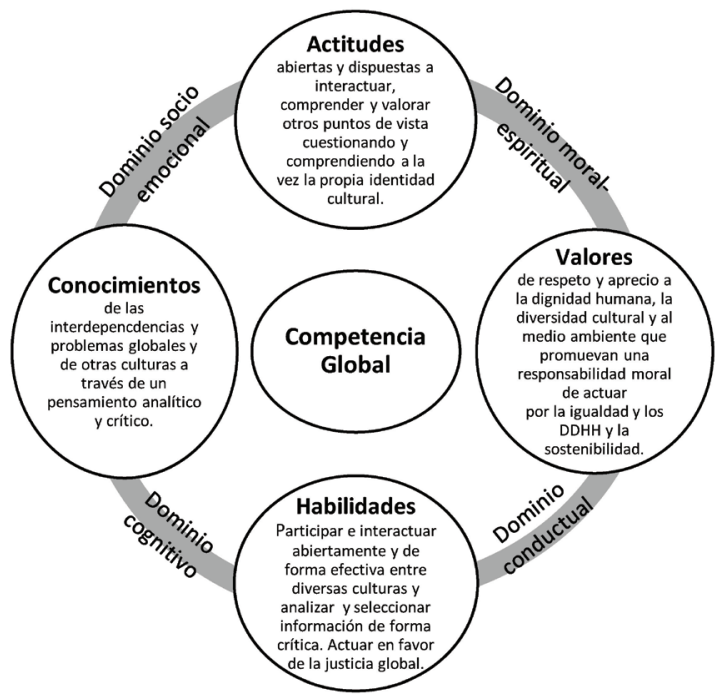

Fuente: Adaptado de Boix-Mansilla \& Jackson, 2011; OCDE, 2018; Reimers, 2010 
Esas dimensiones requieren de un autoconocimiento inicial. Es necesario un trabajo de dentro hacia fuera que está condicionado a una necesaria comprensión de nosotros mismos, del entorno que nos influye, de nuestro lugar en el mundo como base esencial para comprender a los que nos rodean. Aguado (2003) en su descripción de la competencia intercultural señala que las actitudes interculturales «implican disposición o voluntad para cuestionar nuestros propios valores, creencias, y comportamientos, y no asumir que son los únicos posibles o correctos...» (p. 141)

Además de lo ya expuesto, se destaca que la educación intercultural aporta a la competencia global:

- Las actitudes como base necesaria sobre la que desarrollar el resto de las capacidades ligadas a la competencia intercultural y que se traslada al desarrollo de la competencia global según plantean Deardorff (2009) y Cushner (2011).

- El enfoque transformador necesario de ciudadanía global que mejore la democracia, promoviendo la igualdad y justicia social a través de una participación en condiciones de igualdad de todos los actores en la educación (Banks, 2014).

- La hipótesis del contacto desarrollada por Allport (1954) a la hora de diseñar programas que promuevan el desarrollo de la competencia global y que plantea los siguientes principios: a) Los individuos experimentan un estatus igual; b) comparten objetivos comunes; c) existe una cooperación entre grupos; y d) el contacto es supervisado por las autoridades, como los profesores.

\section{LIMITACIONES Y DESAFíOS EN LA CONCEPTUALIZACIÓN DE LA COMPETENCIA GLOBAL}

La pretensión de ser un concepto universal es un desafío que ha acompañado la construcción y evaluación de esta competencia desde siempre. Cuando se diseñó la encuesta sobre conciencia mundial de Barrows (1981) se cuestionaron si las personas que lo formulasen deberían ser miembros de un solo país o de varios para que el instrumento fuese más internacional. Se plantea si eso sería una solución o se estaría cayendo en la habitual categorización de identificar la diversidad con origen nacional, (Aguado, 2009) cuando, además, los actores involucrados en la construcción de marcos de instituciones transnacionales suelen formar parte de coaliciones discursivas de actores que "hablan el mismo idioma" (Nordin \& Sundberg, 2016, p. 320).

Como ya se ha señalado, hay amplia literatura que afirma que existen mecanismos globales de influencia en las políticas educativas locales. Hunter et al. (2006) relacionan la competencia global con el término 'poder blando' acuñado por Joseph S. Nye, que es aquel que defiende el orden mundial de EE. UU. que hace que a otros les guste el sistema estadounidense y lo apoyen por su propia voluntad, frente al 'poder agudo' que tiene que ver con el uso de la violencia, es decir, podría verse como un imperialismo sutil. Esto puede servir como aliciente para proponer más 
interpretaciones culturalmente informadas como la propuesta por Boix-Mansilla \& Wilson (2020).

El hecho de ser un concepto relativamente joven requiere de cautela a la hora de adoptar un marco teórico que aporte una definición y un instrumento de medida válido, pero la introducción de una medida de competencia global en PISA puede dar lugar a que la definición y evaluación planteada por esta institución que obtiene reconocimiento mundial y tiene un fuerte impacto, sea tomada como definitiva y única limitando la investigación en su conceptualización. Varios autores han criticado el marco para la competencia global de PISA 2018, bajo diferentes argumentos, entre ellos; la inclusión solamente de perspectivas occidentales, "El sur nuevamente parece ser irrelevante para el discurso. Los autores de la OCDE del documento final eliminaron voces y fuentes científicas del Sur entre el primer borrador (2014) y el documento final (2018)» (Grotlüschen, 2018, p. 199). Igualmente, Engel et al. (2019) exponen que resulta difícil comparar a nivel internacional e intercultural la adquisición de esta competencia por falta de consenso y transparencia. Plantean que antes de administrar la evaluación, la OCDE debería haber sido más metódica y transparente en cuanto a qué aspectos de la competencia global se consideran universales, y cuáles pueden definirse y medirse mejor a nivel local.

Son precisamente varias autoras participantes en el desarrollo de la evaluación para la competencia global de PISA quienes plantean los desafíos encontrados en la elaboración de una evaluación que es estandarizable y comparable internacionalmente. Para enfrentar el reto de integrar en una misma prueba la enorme variedad de los contextos culturales y geográficos representados en los países participantes, PISA desarrolló dos instrumentos de evaluación; una prueba cognitiva y un cuestionario de contexto. Sälzer \& Roczen (2018) exponen que en la evaluación cognitiva es dificil establecer calificaciones en algunas subdimensiones de correcto o incorrecto para una construcción como competencia global que no es asimilable a dominios basados puramente en la ciencia y que los cuestionarios de contexto tienen un uso frecuente de estereotipos y un sesgo por deseabilidad social. Alguna de esas observaciones puede haber sido la causa de que hasta 30 países decidieran no aplicar la evaluación de esta competencia en 2018 (Sälzer \& Roczen, 2018, p. 6) o que 39 países solo aplicasen el cuestionario de contexto (MEFP, 2020).

Otra crítica en la construcción del marco de PISA es que va cambiando desde un enfoque instrumental de trabajadores con "mentalidad global» a un enfoque en cuestiones globales y sensibilidad intercultural, además de un proceso de alineación ad hoc con los ODS particularmente en el documento final. Y, sobre todo, una adaptación para ser más fácilmente medible que alinea la competencia global con la evaluación ya que, por ejemplo, la comprensión se puede medir más fácilmente que la reflexión crítica (Auld \& Morris, 2019; Sälzer \& Roczen, 2018).

Un aspecto que sí tiene en cuenta el marco de PISA en la medición a través de la autoevaluación del cuestionario del alumno es la competencia lingüística, 
muy vinculada a la comunicación en contextos multiculturales. El desafío que surge respecto a esto, según PISA, (2018) es la dificultad de proteger y reavivar las lenguas aborígenes.

Respecto a las formas de adquirir la competencia global, se encuentra un gran debate, como ya se ha dicho, estudiar en el extranjero es una de las medidas más fomentadas que, a veces, puede no estar al alcance de todas las personas, no garantiza por sí sola el desarrollo de esta competencia y que no tiene por qué ser la única vía para poder desarrollarla (Parkhouse et al., 2016). Autores como Doerr (2020) plantean valorar la potencialidad del alumnado inmigrante como sujetos poseedores de competencia global que pueden contribuir a su desarrollo si se facilita su participación.

Se encuentran por tanto limitaciones y desafíos que tal como explican Sälzer y Roczen (2018) no son irresolubles si se dedica tiempo y esfuerzo en examinar sistemáticamente la construcción del concepto, su desarrollo y medición.

\section{CONCLUSIONES}

La revisión ha mostrado que en la conceptualización de la competencia global ha predominado el enfoque instrumentalista de eficiencia social, aunque sea de manera subyacente frente a un enfoque de re-construccionismo social que suele aparecer en primer plano. El enfoque centrado en el desarrollo de la persona y sus potencialidades es minoritario. No obstante, el lector ha de tener en cuenta que estas afirmaciones surgen de una revisión que permite superar el alcance limitado de revisiones sistemáticas, pero cuenta con una posibilidad de sesgo como debilidad, por lo que se necesitarían estudios más específicos y sistemáticos.

Este trabajo señala las limitaciones inherentes a la conceptualización de la competencia global, e invita a reflexionar sobre qué enfoque se establece en las investigaciones para su desarrollo, cómo se puede evaluar y cómo se puede desarrollar en distintos públicos objetivo. En este sentido, este artículo aporta los cuatro paradigmas (el racionalismo académico, el re-construccionismo social, la eficiencia social, o la ideología curricular centrada en el alumno) como marco para identificar el enfoque en el desarrollo de la competencia global. Además, se concluye que el peso de la educación intercultural en la competencia global es muy significativo, siendo uno de los modelos de educación global, con mayor referencia e influencia en las distintas subdimensiones de esta competencia. Este modelo permite atender los desafíos existentes en la evaluación de la competencia global, así como en los programas para su desarrollo. Una de las claves que aporta este modelo es la importancia del autoconocimiento y las actitudes personales como base fundamental para desarrollar las otras dimensiones. Los profesionales de la educación apoyándose en los principios de la educación intercultural deben aprovechar la diversidad cultural del entorno como medio para desarrollar la competencia global. 
Investigar tanto a nivel teórico como empírico entorno a la definición, medición y desarrollo de la competencia global se trata de un aspecto de relevancia educativa debido a que su adquisición favorece una sociedad que pueda enfrentar los desafíos de la globalización. Por todo lo anterior y tomando la competencia global desde el enfoque del re-construccionismo social y el aprendizaje centrado en el alumno, para seguir investigando y profundizando en el desarrollo y evaluación de esta competencia, proponemos entender la competencia global como un objetivo de aprendizaje permanente que parte de una autorreflexión que facilita a las personas la capacidad y disposición para examinar cuestiones locales, globales e interculturales, comprender y apreciar las interdependencias globales, así como las diferentes perspectivas y visiones del mundo, establecer relaciones positivas en condiciones de igualdad y desde el respeto, con el objetivo de ser agentes transformadores que buscan la justicia social y la sostenibilidad del planeta.

\section{REFERENCIAS BIBLIOGRÁFICAS}

Abdallah-Pretceille, M. (2001). La educación intercultural. Idea Books.

Aguado, T. (2003). Pedagogía Intercultural. McGraw-Hill.

Aguado, T., (2009). El enfoque intercultural como metáfora de la diversidad en educación. En T. Aguado, y M. Del Olmo (Coords.), Educación intercultural: Perspectivas y propuestas (pp. 15-29). Ramón Areces.

Aguado, T., y Mata, P. (2017). Educación intercultural. UNED - Universidad Nacional de Educación a Distancia. https://elibro-net.ubu-es.idm.oclc.org/es/ereader/ ubu/115964?page=1

Allport, G. W. (1954). The nature of prejudice. Addison-Wesley.

American Council on International Intercultural Education. (1996). Educating For The Global Community: A Framework For Community Colleges. https://stanleycenter.org/publications/archive/CC2.pdf

American Council on Education. (1998). Educating for Global Competence. America's Passport to the Future. https://files.eric.ed.gov/fulltext/ED421940.pdf

Auld, E., \& Morris, P. (2019). Science by streetlight and the OECD's measure of global competence: A new yardstick for internationalisation? Policy Futures in Education, 17(6), 677-698. https://doi.org/10.1177/002205741419400302

Banks, J. A. (2014). Diversity, group identity, and citizenship education in a global age. Journal of Education, 194(3), 1-12. https://doi.org/10.1177/002205741419400302

Barrows, et al. (1981). College students' knowledge and beliefs: A survey of global understanding. https://eric.ed.gov/?id=ED215653

Bennet, M. J. (1986). Approach To Training. Intercatural Relations, 10, 179-196.

Boix-Mansilla, V., \& Jackson, A. (2011). Educating for global competence: Preparing our youth to engage the world. Asia Society. https://asiasociety.org/files/book-globalcompetence.pdf 
Boix Mansilla, V., \& Wilson, D. (2020). What is Global Competence, and What Might it Look Like in Chinese Schools? Journal of Research in International Education, 19(1), 3-22. https://doi.org/10.1177/1475240920914089

Castejón, A., y Pàmies, J. (2018). Los agrupamientos escolares: expectativas, prácticas y experiencias. Tendencias Pedagógicas, 32, 49-64. https://doi.org/10.15366/tp2018.32.004

Comisión Europea (CE). (2006) Recomendación del parlamento europeo y del consejo de 18 de diciembre de 2006 sobre las competencias clave para el aprendizaje permanente (2006/962/CE) https://bit.ly/3fck4eT

Cornejo, M. J., y Gómez-Jarabo, I. (2018). Desarrollo de la competencia global en la formación del maestro. El caso de la asignatura Practicum. Innovación educativa, (28), 233-248. https://doi.org/10.15304/ie.28.5361

Curran, K. (n. d.). Global competencies that facilitate working effectively across cultures.

Cushner, K. (2011). Intercultural research in teacher education: An essential intersection in the preparation of globally competent teachers. Action in Teacher Education, 33(5-6), 601-614. https://doi.org/10.1080/01626620.2011.627306

Deardorff, D. K. (Ed). (2009). Intercultural competence. SAGE Publications.

Delors, J. et al. (1996). La educación encierra un tesoro. Informe a la UNESCO de la Comisión Internacional sobre la educación para el siglo XXI. Santillana.

Deng, Z., \& Luke, A. (2008). Subject Matter: Defining and Theorizing School Subjects. The SAGE handbook of curriculum and instruction, 66-87. https://doi.org/10.4135/9781412976572.n4

Doerr, N. M. (2020) 'Global competence' of minority immigrant students: hierarchy of experience and ideology of global competence in study abroad. Discourse: Studies in the Cultural Politics of Education, 41(1), 83-97. https://doi.org/10.1080/01596306.2018.1462147

Engel, L. C., Rutkowski, D., \& Thompson, G. (2019). Toward an international measure of global competence ? A critical look at the PISA 2018 framework. Globalisation, Societies and Education, 17(2), 117-131. https://doi.org/10.1080/14767724.2019.1642183

García, M. E. (2008). La evaluación por competencias en la educación superior. Profesorado. Revista de Currículum y Formación Del Profesorado, 12(3), 1-16. https://bit.ly/352gK2h

García-Beltrán, E., Bueno, A., y Teba, E. (2019). Uso del modelo CCR en la investigación sobre competencia global. En Larragueta, M (coord), Educación y transformación social $y$ cultural (pp. 361-380) Universitas. https://bit.ly/3naeFYe

Goren, H., \& Yemini, M. (2017). Global citizenship education redefined-A systematic review of empirical studies on global citizenship education. International Journal of Educational Research, 82, 170-183. https://doi.org/10.1016/j.ijer.2017.02.004

Grant, M. J., \& Booth, A. (2009). A typology of reviews: an analysis of 14 review types and associated methodologies. Health information \& libraries journal, 26(2), 91-108.

Guirao Goris, Silamani J. A. (2015). Usefulness and types of literature review. Ene, 9(2). https:// doi.org/10.4321/S1988-348X2015000200002

Grotlüschen, A. (2018). Global competence-Does the new OECD competence domain ignore the global South? Studies in the Education of Adults, 50(2), 185-202. https://doi.org/10 .1080/02660830.2018.1523100

Hajisoteriou, C., y Angelides, P. (2016). The Globalisation of Intercultural Education. The Politics of Macro-Micro Integration. Palgrave-Macmillan. 
Hajisoteriou, C., \& Angelides, P. (2020). Examining the nexus of globalisation and intercultural education: theorising the macro-micro integration process. Globalisation, Societies and Education, 18(2), 149-166. https://doi.org/10.1080/14767724.2019.1693350

Hanvey, R. (1975). An Attainable Global Perspective. Center for Global Perspectives.

Heras, R., Vila, J., y Medir, R. M. (2019). Estrategias educativas para comprender y actuar en el mundo actual. Papeles de Trabajo Sobre Cultura, Educación y Desarrollo Humano, 15(3), 73-83. https://bit.ly/3mTkcm7

Hernández, R., Fernández, C., y Baptista, P. (2010). Metodología de la investigación. McGrawHill. $5^{\mathrm{a}}$ ed.

Hunter, W. (2004). Knowledge, skills, attitudes, and experiences necessary to become globally competent. Tesis para doctorado. Lehigh University. https://bit.ly/38f2sNS

Hunter, B., White, G. P., \& Godbey, G. C. (2006). What does it mean to be globally competent? Journal of Studies in International education, 10(3), 267-285. https://doi. org/10.1177/1028315306286930

Kim, J. J. (2019). Conceptualising global competence: situating cosmopolitan student identities within internationalising South Korean Universities. Globalisation, Societies and Education, 17(5), 622-637. https://doi.org/10.1080/14767724.2019.1653755

Lambert, R. (1993). Educational Exchange and Global Competence. International Conference on Educational Exchange. Council on International Educational Exchange. https://files. eric.ed.gov/fulltext/ED368275.pdf

Leal, M. (2016). Dinámicas de identidad y educación intercultural en contextos urbanos. Opción, 32(11),787-807 https://www.redalyc.org/pdf/310/31048902046.pdf

Ministerio de Educación y Formación Profesional [MEFP] (2020). Informe español, versión preliminar. Competencia global PISA 2018. Secretaría General Técnica. https://bit.ly/3mVhpbT

Nordin, A., \& Sundberg, D. (2016). Travelling concepts in national curriculum policy-making: the example of competencies. European Educational Research Journal, 15(3), 314-328. https://doi.org/10.1177/1474904116641697

OCDE. (2005). The definition and selection of key competencies [Executive Summary] https:// www.oecd.org/pisa/35070367.pdf

OCDE. (2014). PISA 2018 Framework plans: 38th meeting of the PISA governing board. Statistics, December, 1-13. https://bit.ly/3k1x5IQ

OCDE. (2018). Preparar a nuestros jóvenes para un mundo inclusivo y sostenible. PISA 2018 Marco de Competencia Global. Estudio PISA. https://bit.ly/313UNWb

Olson, C., \& Kroeger, K. R. (2001). Global Competency and Intercultural Sensitivity. Journal of studies in international education, 5(2), 116-137. https://doi.org/10.1177/102831530152003

Ortiz-Revilla, J., Adúriz-Bravo, A., y Greca, I. M. (en prensa). Conceptualización de las competencias: Revisión sistemática de su investigación en educación primaria. Profesorado. Revista de Currículum y Formación Del Profesorado. https://bit.ly/3mX1Ctj

Parkhouse, H., Tichnor-Wagner, A., Cain, J. M., \& Glazier, J. (2016). "You don't have to travel the worldy: accumulating experiences on the path toward globally competent teaching. Teaching Education, 27(3), 267-285. https://doi.org/10.1080/10476210.2015.1118032 
Reimers, F. (2010). Educating for Global Competency. En J. Cohen \& M. Malin (Eds.), International perspectives on the goals of universal basic and secondary education. (pp.183202). Routledge.

Sälzer, C., E Roczen, N. (2018). Assessing Global Competence in PISA 2018: Challenges and Approaches to Capturing a Complex Construct. International journal of development education and global learning, 10(1), 5-20. https://doi.org/10.18546/IJDEGL.10.1.02

Santander, P. (2011). Por qué y cómo hacer Análisis de Discurso. Cinta de Moebio, (41), 207-224. https://doi.org/10.4067/S0717-554X2011000200006

Schiro, M. S. (2013). Curriculum theory: conflicting visions and enduring concerns. Sage Publications.

Schmidt, V. A. (2015). Discursive institutionalism: understanding policy in context. In F. Frank Fischer, D. Torgerson, A. Durnovà, \& M. Orsini (Eds.), Handbook of critical policy studies (pp. 187-205). Edward Elgar Publishing.

Tahirsylaj, A., \& Sundberg, D. (2020). The unfinished business of defining competences for 21st century curricula-a systematic research review. Curriculum Perspectives, 40(2), 131-145. https://doi.org/10.1007/s41297-020-00112-6

UNESCO. (2015). Declaración de Incheon para la Educación 2030. https://bit.ly/2IJkeyi

Wilson, M. S., \& Dalton, M. A. (1997). Understanding the demands of leading in a global environment: A first step. Issues and Observations, 17(1/2), 12-14. 